\title{
Nck1 is a critical adaptor between proatherogenic blood flow, inflammation, and atherosclerosis
}

\author{
Mary Wines-Samuelson, Sayantani Chowdhury, and Bradford C. Berk \\ Department of Medicine, Aab Cardiovascular Research Institute, and University of Rochester Neurorestoration Institute, University of Rochester School of Medicine and Dentistry, Rochester, New York, USA.
}

\begin{abstract}
Atherosclerosis is an inflammatory condition of the arteries that has profound incidence and increasing prevalence. Although endothelial cells detect changes in blood flow, how endothelial activation contributes to atherogenic inflammation is not well understood. In this issue of the $J C I$, Alfaidi et al. used mouse models to explore flow-induced endothelial activation. The authors revealed a role for Nck1 and a specific activator of the innate immune response, the downstream interleukin receptor-associated kinase-1 (IRAK-1) in NF-KB-mediated inflammation and atherosclerosis susceptibility. These results link disturbed blood flow to NF- $\mathrm{KB}-$ mediated inflammation, which promotes atherosclerosis, and provide Nck1 as a potential target for the treatment of atherosclerosis.
\end{abstract}

ECs exposed to d-flow is consistent with human data from a large 2018 GWAS study, which identified Nck1 as a coronary artery disease susceptibility locus (2).

\section{Noncompensatory roles of Nck1 and Nck2 in inflammation}

Nck1 and Nck2 are paralogous adaptor proteins with essential and initially redundant functions via the EC lineage in embryonic blood vessel and heart development (3). Originally, these adaptors were thought primarily to connect receptor tyrosine kinases (RTKs) to actin cytoskeleton regulators $(4,5)$. In Alfaidi et al., the authors demonstrate that in Nck1-depleted ECs under d-flow, NF- $\mathrm{B}$ activation is attenuated; in contrast, Nck2 depletion has little effect (1). These data are consistent with the authors' previous work, which identified a requirement for Nck1, but not Nck2, in oxidative stress-induced EC permeability (6) and in recruitment of p21-activated kinase-2 (PAK2) to PECAM-1 (7). Thus, a major contribution of the authors' present work is their identification of Nck1 as a mediator of d-flow-induced endothelial inflammation, highlighting a clearly nonredundant functional difference between Nck1 and Nck2.

A model that incorporates the major findings of Alfaidi et al. (Figure 1) shows that under s-flow, there is no signaling by the Nck1/IRAK-1/NF-кB p65 pathway (1). Under s-flow, the flow sensor, which is probably an RTK, likely remains unactivated. Alternatively, the RTK binds and inhibits Nck1. In d-flow, the RTK binds Nck1 and activates the Nck1 proinflammatory pathway, and/or Nck1 is released from the membrane.

Nck1, d-flow, atherosclerosis, and NF- $\kappa$ B activation

Nck1 regulates at least 3 key pathways involved in progression of atherosclerosis. First, previous work by the Orr labo- 
A

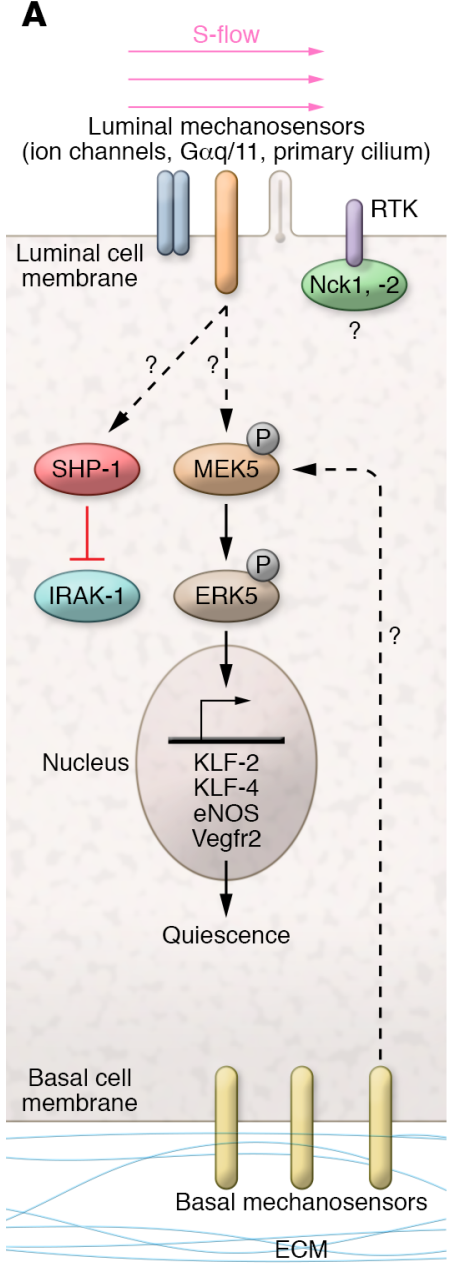

B

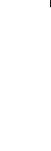

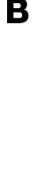
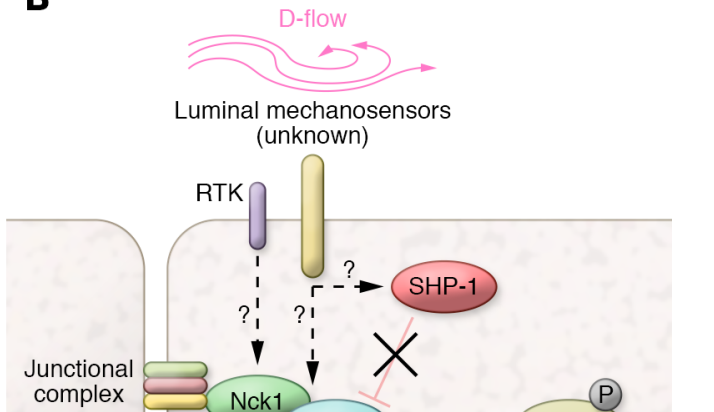

Figure 1. Flow-dependent regulation of Nck1 adaptor and IRAK-1. (A) In endothelial cells (ECs) under steady laminar flow (s-flow), homeostatic mechanisms are activated: flow mechanosensors include ion channels (e.g., TRPV4, TRPC1, Piezo1/2), G protein-coupled receptors and heterotrimeric G proteins $(\mathrm{G} \alpha \mathrm{q} / 11)$, and the primary cilium; the junctional complex (containing Vegfr2/Flk-1, VE-cadherin, and PECAM-1) detects EC-EC junctions, and the basal mechanosensors (integrin receptors) linked to the extracellular matrix detect adhesion. Receptor tyrosine kinases (RTKs) present in ECs are EGFR, Fgfr-1, and Vegfr2; the SH2 domains of Nck1 and 2 may bind to RTKs. In s-flow, downstream of flow sensors and RTK, the kinases MEKK2/3 activate MEK5 and phosphorylate ERK5, which activates transcription factors KLF-2/KLF-4, leading to increases in eNOS and VEGFR2 to promote quiescence. (B) A simplified diagram of ECs under disturbed flow (d-flow) focuses on the Nck/IRAK-1 pathway to inflammation. An unknown mechanosensor signals to promote the binding of Nck1 to IRAK-1 under d-flow, which may involve Nck1 binding to PECAM-1 (5). Nck1 was shown previously to bind to the kinase PAK2 (6). The direct interaction with Nck1 leads to IRAK-1 phosphorylation, possibly by PAK2, to become active. p-IRAK-1 then phosphorylates IKB kinase (IKK), which in turn phosphorylates IKB and p65; IKB releases $p 65$, which translocates to the nucleus to upregulate proinflammatory gene expression (e.g., VCAM1, ICAM1, IL-1, IL-6, and MCP-1).

ratory demonstrated that Nck1 binds to PECAM-1 in the adhesion junction complex to regulate vascular permeability $(6$, 7). Vascular permeability is increased by d-flow, allowing uptake of low-density lipoproteins (LDLs) and other plasma components that stimulate inflammation in the vessel wall $(8,9)$. These data provide a direct link between Nck1 binding to PECAM-1 and LDL deposition in the intima (10). Second, both Chen et al. and Alfaidi et al. demonstrated that Nck1, but not Nck2, increases oxidative stress in ECs, which decreases nitric oxide, promoting EC dysfunction (11). Third, Nck1 is a major regulator of d-flow-mediated NF- $\mathrm{kB}$ signaling, as shown by Alfaidi et al., who demonstrated that knockdown of Nck1/2, or loss of Nck1 alone, in ECs under d-flow attenu- ated NF- $\mathrm{KB}$ signaling and $\mathrm{EC}$ activation in vitro (1). Furthermore, the authors found in ligated carotids (a model for d-flow) of Nck1-KO and EC-Nck1/2-DKO mice that Nck1 was specifically required for EC activation, as measured by decreased expression of proinflammatory VCAM-1, ICAM-1, and Mac-2 in d-flow.

Together, the present results linking d-flow to Nck1, and Nck1 to IRAK-1 in the

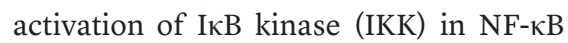
signaling, define a new pathway of flow-mediated inflammation. Because IRAK-1 phosphorylation/activation in ECs depends on the presence of Nck1, a major contribution by Alfaidi and coauthors was to show Nck1 as a pivotal point in this pathway. This studyemphasizes that multifunctional adaptors represent key signaling hubs that participate in transducing both homeostatic EC function (quiescence) and stimulation of multiple pathologic events (inflammation, permeability, and oxidative stress) $(1,12)$.

\section{Inflammation and NF- $\kappa B$ activation by IRAK-1/IRAK-4}

IRAK-4 is the endogenous activator of IRAK-1, a critical kinase upstream of NF- $\kappa B$ activation (13). Following activation of IL-1R or TLR, MyD88 binds to the receptor complex, and then to IRAK-4, which recruits and phosphorylates IRAK-1 to activate it in the myddosome complex. Subsequent recruitment of TRAF6 leads to activation of IKK, p65 nuclear translocation, and expression of the proinflammatory phenotype $(14,15)$. IRAK-1 activity is directly inhibited by Shp-1 phosphatase, which in turn suppresses NF-KB activation (16).

The findings by Alfaidi et al. that (a) Nck1 and p-IRAK-1 interact under shear stress, and (b) p-IRAK-1 levels increase in ECs following carotid ligation, place IRAK-1 in the d-flow-response pathway in ECs in vivo (1). Additionally, loss of IRAK-1 in ECs decreases NF- $\mathrm{KB}$ activation and downstream proinflammatory expression of VCAM-1 and ICAM-1, which clearly identifies IRAK-1 as a downstream effector of Nck1 in d-flow-mediated EC inflammation (1). It would be interesting to observe how the effects of loss of EC IRAK- 1 compare with loss of EC Nck1 in d-flow and in a model of atherosclerosis. Is IRAK- 1 the only signaling factor between Nck1 and 
$\mathrm{NF}-\kappa \mathrm{B}$ activation in flow-responsive signaling, and what are the specific upstream regulators for IRAK-1 in EC flow signaling?

\section{Future directions for endothelial} Nck adaptors and IRAK-1

Although the Alfaidi et al. study clearly places endothelial Nck1 upstream of IRAK-1 in the d-flow-response pathway to inflammation and atherosclerosis susceptibility (Figure 1), there are several questions that remain unanswered (1). What is the flow-sensing receptor upstream of Nck1? Is PECAM-1 relaying the signal to Nck1 in d-flow, or some other tyrosine-phosphorylated mechanosensor? The known interaction of Ncks with RTKs IL-1R, IRS-1, and EGFR points to their potential importance in the Nck-regulated d-flow-mediated pathway. Further, the study of Nck interactors by Jacquet et al. should provide candidates for future research (17). The relative contribution of each Nck to different d-flow-responsive proinflammatory pathways remains to be elucidated. In addition, does Nck1 promote EC inflammation by suppressing s-flow signaling (e.g., Klf2/Klf4 expression) in parallel with its proatherogenic d-flow signaling? Alternatively, does Nck1 serve different roles in s-flow versus d-flow? Nck1 is known to regulate EC polarity and coordinate VEGF-A/Slit2 signaling (18), indicating a vital function in nonactivated ECs. And finally, are other adaptors playing critical roles in mediating the integration of s-flow and/or d-flow signals? Future studies to identify key integration points between signaling pathways will allow us to more clearly understand the coordinated responses of ECs to atherogenic stimuli.

\section{Acknowledgments}

$\mathrm{BCB}$ is supported in part by funds from the NIH (R01 HL049192, R01 HL134910, and R01 HL140598).

Address correspondence to: Bradford C. Berk, University of Rochester Medical Center, 601 Elmwood Avenue, Box URNI, Rochester, New York 14642, USA. Phone: 585.276.7418; Email: bradford_berk@ urmc.rochester.edu

1. Alfaidi M, Acosta CH, Wang D, Traylor JG, Orr AW. Selective role of Nck1 in atherogenic inflammation and plaque formation. J Clin Invest. 2020;130(8):4331-4347.

2. van der Harst P, Verweij N. Identification of 64 novel genetic loci provides an expanded view on the genetic architecture of coronary artery disease. Circ Res. 2018;122(3):433-443.

3. Clouthier DL, Harris CN, Harris RA, Martin CE, Puri MC, Jones N. Requisite role for Nck adaptors in cardiovascular development, endothelial-to-mesenchymal transition, and directed cell migration. Mol Cell Biol. 2015;35(9):1573-1587.

4. Buday L, Wunderlich L, Tamás P. The Nck family of adapter proteins: regulators of actin cytoskeleton. Cell Signal. 2002;14(9):723-731.

5 . Frese $S$, et al. The phosphotyrosine peptide binding specificity of Nck1 and Nck2 Src homology 2 domains. J Biol Chem. 2006;281(26):18236-18245.

6. Alfaidi M, Acosta CH, Lindquist JM, Cockerham ED, and Orr AW. The differential roles of the adaptor proteins Nck1 and Nck2 in shear stress-induced endothelial activation [preprint]. https://doi.org/10.1101/668129. Posted on bioRxiv June 11, 2019.

7. Chen J, et al. Recruitment of the adaptor protein Nck to PECAM-1 couples oxidative stress to canonical NF- $\mathrm{B}$ signaling and inflammation. Sci Signal. 2015;8(365):ra20.

8. Fry DL, Herderick EE, Johnson DK. Local intimal-medial uptakes of ${ }^{125} \mathrm{I}$-albumin, ${ }^{125} \mathrm{I}$-LDL, and parenteral Evans blue dye protein complex along the aortas of normocholesterolemic minipigs as predictors of subsequent hypercholesterolemic atherogenesis. Arterioscler Thromb 1993;13(8):1193-1204

9. Soulis JV, Fytanidis DK, Papaioannou VC, Giannoglou GD. Wall shear stress on LDL accumulation in human RCAs. Med Eng Phys. 2010;32(8):867-877.

10. Mundi S, et al. Endothelial permeability, LDL deposition, and cardiovascular risk factors-a review. Cardiovasc Res. 2018;114(1):35-52.

11. Gimbrone MA, García-Cardeña G. Endothelial cell dysfunction and the pathobiology of atherosclerosis. Circ Res. 2016;118(4):620-636.

12. Naudin C, Chevalier C, Roche $\mathrm{S}$. The role of small adaptor proteins in the control of oncogenic signalingr driven by tyrosine kinases in human cancer. Oncotarget. 2016;7(10):11033-11055.

13. Bhaumik D, Scott GK, Schokrpur S, Patil CK, Campisi J, Benz CC. Expression of microRNA-146 suppresses NF-kappaB activity with reduction of metastatic potential in breast cancer cells. Oncogene. 2008;27(42):5643-5647.

14. Song KW, et al. The kinase activities of interleukin-1 receptor associated kinase (IRAK)-1 and 4 are redundant in the control of inflammatory cytokine expression in human cells. Mol Immunol. 2009;46(7):1458-1466.

15. Singer JW, Fleischman A, Al-Fayoumi S, Mascarenhas JO, Yu Q, Agarwal A. Inhibition of interleukin-1 receptor-associated kinase 1 (IRAK1) as a therapeutic strategy. Oncotarget. 2018;9(70):33416-33439.

16. An $\mathrm{H}$, et al. Phosphatase SHP-1 promotes TLR- and RIG-I-activated production of type I interferon by inhibiting the kinase IRAK1. Nat Immunol. 2008;9(5):542-550.

17. Jacquet K, Banerjee SL, Chartier FJM, Elowe $\mathrm{S}$, Bisson N. Proteomic analysis of NCK1/2 adaptors uncovers paralog-specific interactions that reveal a new role for NCK2 in cell abscission during cytokinesis. Mol Cell Proteomics. 2018;17(10):1979-1990.

18. Dubrac A, et al. Targeting NCK-mediated endothelial cell front-rear polarity inhibits neovascularization. Circulation. 2016;133(4):409-421. 